\title{
OVULATION-INDUCING ACTION OF CLOMIPHENE CITRATE IN THE RAT
}

\author{
F. DÖGKE \\ Institute of Experimental Endocrinology, Charité, Humboldt University, \\ Berlin, Germany
}

(Received 3rd September 1968)

\begin{abstract}
Summary. Corpus luteum formation was induced by s.c. injections of clomiphene citrate in female rats made anovulatory by postnatal androgenization, continuous light or electrolytic lesions in the medial pre-optic-suprachiasmatic region of the hypothalamus.
\end{abstract}

Clomiphene (MRL-41), defined chemically as 1-[ $p$-( $\beta$-diethyl-aminoethoxy)phenyl]1,2-diphenyl-2-chloroethylene, is now widely used for the induction of ovulation in women. However, the mechanism of action of this non-steroidal agent has not been elucidated. This may be due in part to the fact that since the studies of Holtkamp, Greslin, Root \& Lerner (1960), Holtkamp, Davis \& Rhoads (1961) and Barnes \& Meyer (1962) it has been generally accepted that clomiphene does not stimulate but rather inhibits gonadotrophin secretion and ovulation in adult female rats. Thus, adequate experiments in this species could not be performed.

On the assumption that the apparent difference of response in rats and women to clomiphene might be due to the use of inappropriate experimental animals, experiments were performed on female rats which were believed to be more comparable to anovulatory women reacting to clomiphene.

Newborn female Wistar rats were injected subcutaneously with 5,10 or $100 \mu \mathrm{g}$ testosterone propionate (TP) on the 3rd day of life. Three weeks later the animals were spayed and their ovaries were autotransplanted into their eyes according to the method of Browning \& Guzman (1967). The grafts were inspected weekly and at the time after puberty when no corpora lutea were visible $2 \mathrm{mg}$ clomiphene citrate/ $\mathrm{kg}$ body weight were injected daily s.c. for 5 days. Each dose was suspended in $0.2 \mathrm{ml}$ olive-oil, the controls receiving oil only.

Female Sprague-Dawley rats weighing 150 to $180 \mathrm{~g}$ were exposed to continuous illumination for 60 to 70 days and were then subjected to laparotomy. Fragments of those ovaries which contained no freshly formed corpora lutea were autotransplanted into the anterior chamber of both eyes. Beginning on the 20th day after surgery, rats which showed no corpus luteum formation during this time were daily injected with 5 or $10 \mathrm{mg}$ clomiphene citrate/ $\mathrm{kg}$ for 5 days. Intra-ocular ovarian grafts were performed in this and the first 


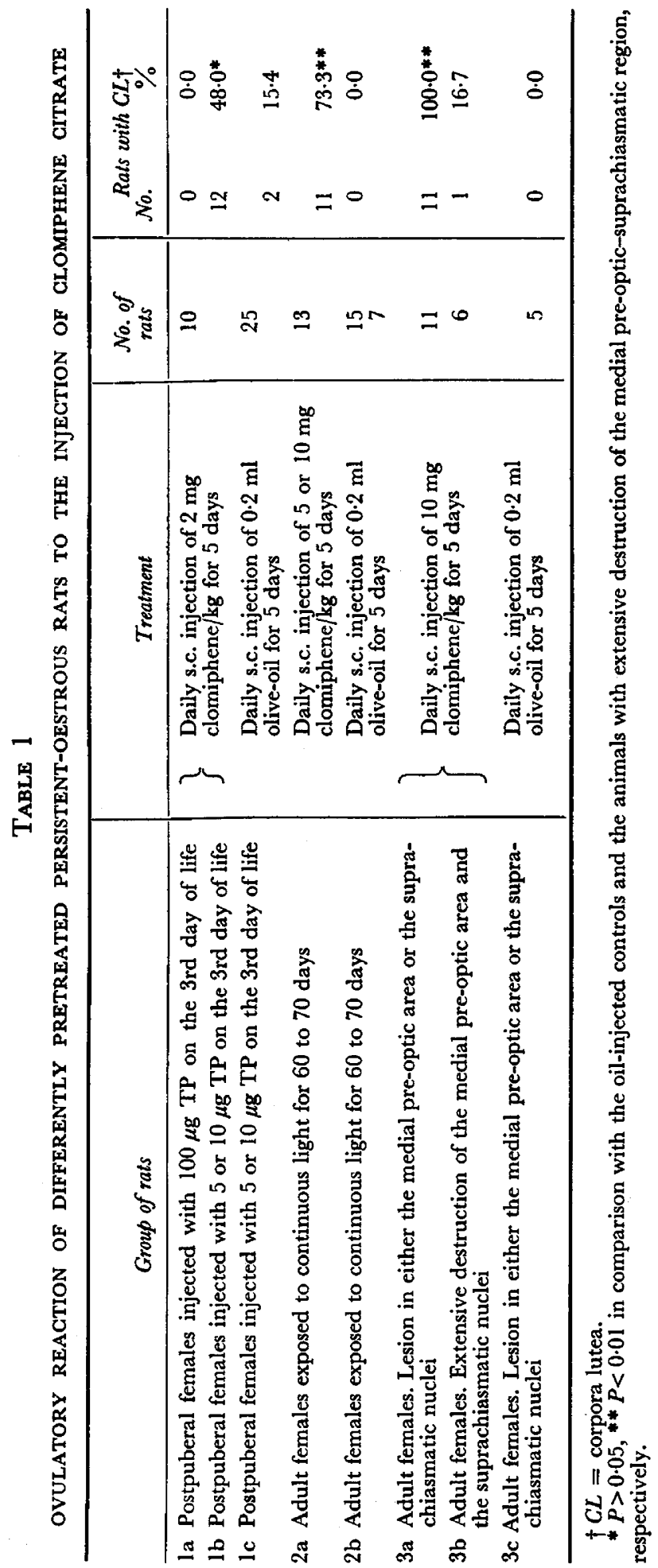


experiment to find out the beginning of the true polyfollicular state. Preliminary studies had shown that the formation of corpora lutea stopped at different times after the beginning of permanent vaginal cornification.

In the last experiment, bilateral electrolytic lesions $(2 \mathrm{~mA} / 7 \mathrm{sec})$ were placed in the medio-basal anterior hypothalamus of cyclic female Sprague-Dawley rats weighing 180 to $220 \mathrm{~g}$. Animals exhibiting at least 4 weeks of persistent vaginal oestrus after surgery were subjected to laparotomy to establish the absence of corpora lutea and were then injected daily with $10 \mathrm{mg}$ clomiphene citrate $/ \mathrm{kg}$ for 5 days. Autopsies were carried out on all rats on the 8th day after beginning the clomiphene treatment. The intra-ocular ovarian grafts of the androgenized and light-stimulated animals, and the ovaries and hypothalami of the lesioned rats were serially sectioned and stained with haematoxylin and eosin to study corpus luteum formation and the localization of the electrolytic lesions.

The results represented in Table 1 show that clomiphene citrate induced ovulation in female rats made anovulatory by postnatal injection of low doses of TP, continuous light or small hypothalamic lesions, the reliability of the effect increasing in this sequence. The last experiment, in which ovulation occurred in all treated rats with isolated destruction of either the medial pre-optic area or the suprachiasmatic nuclei, suggests that the cyclic gonadotrophin-controlling function of the medial pre-optic anterior hypothalamic region must be preserved to a certain degree in anovulatory rats that respond to the clomiphene treatment. This condition may not only be found in persistent-oestrous rats with small anterior hypothalamic lesions, but it may also occur temporarily in postpuberal rats that have been exposed to continuous light or to neonatal treatment with low doses of TP (Lawton \& Schwartz, 1967; Swanson \& Van der Werff ten Bosch, 1964). After postnatal androgenization with $100 \mu \mathrm{g}$ (Group 1a) or 1.25 mg TP (Roy, Greenblatt \& Mahesh, 1964) an almost complete inactivity of the cyclic gonadotrophin-controlling centre may exist already shortly after puberty and may prevent the induction of ovulation by clomiphene in these rats.

The clomiphene citrate used in this study was generously supplied by Professor Garten of the Wm. S. Merrell Company.

\section{REFERENGES}

BARnes, L. E. \& MEYeR, R. K. (1962) Effects of ethamoxy-triphetol, MRL-37, and clomiphene on reproduction in rats. Fert. Steril. 13, 472.

BRowning, H. C. \& Guzman, R. (1967) Intraocular ovarian isografts in female rats and their response to gonadotropins and to pituitary isografts. Endocrinology, 81, 1311.

Holtkamp, D. E., Davis, R. H. \& Rhoads, J. E. (1961) Effect of chloramiphene on fertility and ovulation. Fedn Proc. Fedn Am. Socs exp. Biol. 20, 419.

Holtramp, D. E., Greslin, J. G., Root, C. A. \& Lerner, L. J. (1960) Gonadotrophin inhibiting and antifecundity effects of chloramiphene. Proc. Soc. exp. Biol. Med. 105, 197.

Lawton, I. E. \& Schwartz, N. B. (1967) Pituitary-ovarian function in rats exposed to constant light: A chronological study. Endocrinology, 81, 497.

Roy, S., Greenblatt, R. B. \& MAHESh, V. B. (1964) Effects of clomiphene and intrasplenic ovarian autotransplantation on the anovulatory cystic ovaries of rats having androgen-induced persistent estrus. Fert. Steril. 15, 310.

Swanson, H. E. \& VAN DER Werff ten Bosch, J. J. (1964) The "early-androgen" syndrome; its development and the response to hemispaying. Acta endocr., Copenh. 45, 1. 